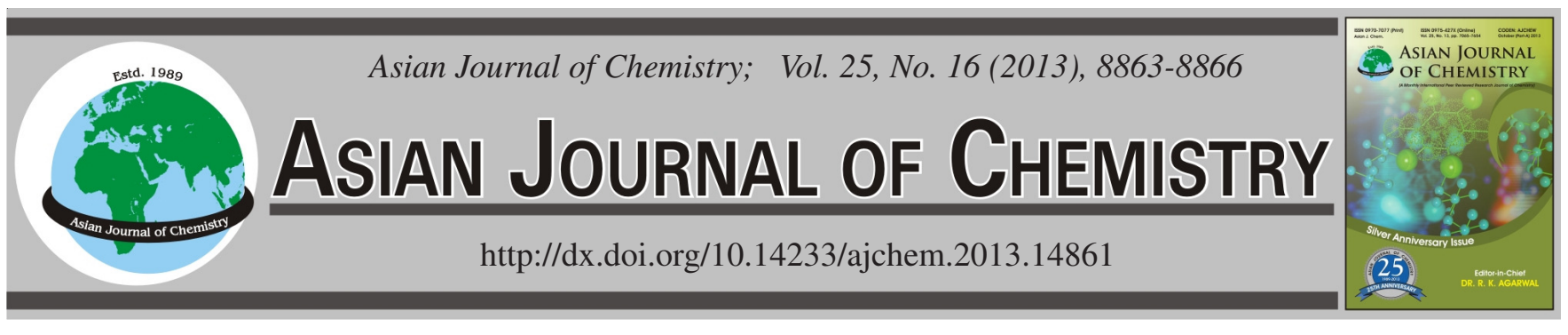

\title{
Antioxidation Mechanisms of Catechin, Epicatechin and Quercetin Using Linear Solvation Energy Relationship
}

\author{
Y. JIN ${ }^{1}$, Y.D. CHENG ${ }^{1}$, K.H. Row ${ }^{2}$, Y.S. JiN ${ }^{3}$, Y.H. XUAN ${ }^{4}$ and M.J. JiN ${ }^{5}$ **
}

${ }^{1}$ College of Food Science and Technology, Shanghai Ocean University, Shanghai 201306, P.R. China

${ }^{2}$ Department of Chemistry and Chemical Engineering, Inha University, Incheon 402-751, Republic of Korea

${ }^{3}$ College of Bioscience and Biotechnology, Yangzhou University, Yangzhou 225009, P.R. China

${ }^{4}$ Guangling College, Yangzhou University, Yangzhou 225009, P.R. China

${ }^{5}$ Department of Forestry Science, Agricultural College of Yanbian University, Yanji 133002, Jilin Province, P.R. China

*Corresponding author: Fax: +86 433 2435600; Tel: +86 433 2435556; E-mail: jinmingji@ybu.edu.cn

(Received: 23 November 2012;

Accepted: 5 September 2013)

AJC-14068

\begin{abstract}
The fundamental chemical interactions governing the anti-oxidations such as ferric reducing power (FRAP) assay, two methods based on the ability to scavenge the $\mathrm{ABTS}^{-+}$radical cation, scavenging of the stable $\mathrm{DPPH}^{*}$ radical and of authentic peroxynitrite $\left(\mathrm{ONOO}^{-}\right)$of three polyphenols such as catechin, epicatechin and quercetin were investigated using linear solvation energy relationship (LSER) model. The ability of the LSER to account for the chemical interactions underlying polyphenols antioxidation was shown. A comparison of predicted and experimental antioxidation activities suggests that LSER formalism is able to reproduce adequately the experimental antioxidation activities of the three polyphenols studied in the different experimental conditions investigated. The predicted antioxidation activities by the LSER model have good agreement with experimental data in the employed conditions.
\end{abstract}

Key Words: Antioxidation mechanism, Catechin, Epicatechin, Quercetin, Linear solvation energy relationship.

\section{INTRODUCTION}

Biological free radical reactions are involved in the reduction of molecular oxygen to yield reactive oxygen species (ROS) such as the superoxide anion and hydroxyl radical. Various diseases such as cancer, liver disease and diabetes may reduce the number of these radicals and reactive oxygen species may be essential for cellular functions such as ingestion of bacteria and redox regulation of signal transduction. These reactive oxygen species cause destructive and damage to the components of a cell, such as lipids, proteins and DNA. Moreover, lipid peroxides promote the formation of additional free radical in a type of chain reaction. ${ }^{1}$ Several methods have been proposed to evaluate the antioxidant activity of vegetal extracts and pure compounds and it is accepted that this effect depends on the environmental conditions and procedures. In vitro assays for the free radical scavenging capacity are commonly based on the inactivation of stable synthetic radicals, such as the 2,2-diphenyl-1-picrylhydrazyl (DPPH*), first envisaged by Blois. Another interesting approach is the study of the peroxynitrite $\left(\mathrm{ONOO}^{-}\right)$scavenging capacity. Peroxynitrite is nowadays considered one of the most relevant radical generator involved in pathophysiological and toxicological processes. This anion is a product of the reaction between nitric oxide and superoxide and is a potent and versatile oxidant. Its importance in biological systems is based on its powerful ability to react with almost all classes of biomolecules. In fact, while it is relatively stable under basic conditions, at physiological conditions it forms two radicals $\left(\mathrm{NO}_{2}{ }^{*}\right.$ and $\left.\mathrm{OH}^{*}\right)$ that induce lipid peroxidation, disruption of cellular structures, inactivation of enzymes and ion channels through protein oxidation and nitration and DNA damages. All these actions contribute to the onset and maintenance of pathologies such as atherosclerosis, neurodegenerative diseases and cardiovascular disorders. Scavengers of these deleterious radicals and compounds able to prevent the consequences of their reactivity can contribute to the maintenance of health or healing processes $^{2}$.

Flavonoids are plant pigments that are synthesized from phenylalanine, generally display marvelous colours known from flower petals, mostly emit brilliant fluorescence when they are excited by UV light and are ubiquitous to green plant cells. The flavonoids are used by botanists for taxonomical classification. They regulate plant growth by inhibition of the exocytosis of the auxin indolyl acetic acid, as well as by induction of gene expression and they influence other biological cells in numerous ways. Flavonoids inhibit or kill many 
bacterial strains, inhibit important viral enzymes, such as reverse transcriptase and protease and destroy some pathogenic protozoans. Yet, their toxicity to animal cells is low. Flavonoids are major functional components of many herbal and insect preparations for medical use, e.g., propolis (bee's glue) and honey, which have been used since ancient times. The daily intake of flavonoids with normal food, especially fruit and vegetables, is $1-2 \mathrm{~g}$. Modern authorized physicians are increasing their use of pure flavonoids to treat many important common diseases, due to their proven ability to inhibit specific enzymes, to simulate some hormones and neurotransmitters and to scavenge free radicals ${ }^{3}$. Catechins and quercetin are major polyphenols in many plant foods that have been related to health promotion. In the human organism they are largely metabolized to different metabolites, which are further found in plasma and should contribute to the biological effects associated to the intake of the parent compounds. An important step in quercetin and catechins metabolism is the O-methylation of the catechol group, which can be expected to have an effect on their antioxidant and scavenging properties ${ }^{4}$.

Quantitative structure-retention relationships (QSRRs), one of the more commonly used empirical approaches, generally take the form of multiple linear regression relationships. Quantitative structure-retention relationships describe specific chromatographic retention phenomena and offer good predictability within a compound class on a given stationary phase. Unfortunately, the relationships obtained cannot be reliably extended to other chemical families or generalized to behaviour on other stationary phases ${ }^{5}$. Quantitative structure-activity relationship (QSAR) theory was developed in the form of a linear solvation energy relationship (LSER) by Kamlet et al. ${ }^{6}$, who used a multi-parameter approach to describe configurational properties in terms of a solute cavity in a solvent and the solventsolute interactions. Since then, the theory has been adapted to other aspects of chemistry including reaction rates, toxicity, fluorescence lifetimes and others. LSER methods involve the application of solvent parameters in linear or multiple-linear regression formulations to express solvent effects for property and reactivity prediction. Properties of mixtures, such as chromatographic retentions, water-octanol partition coefficients and solubilities have been successfully predicted using LSER methods ${ }^{7,8}$. In this study, the antioxidation activities of three polyphenols (catechin, epicatechin and quercetin) were investigated using linear solvation energy relationship.

\section{EXPERIMENTAL}

Estimation of linear solvation energy relationship: Antioxidation activities were referenced for the three polyphenols used in this study and the system constants were calculated by multiple linear regressions using Origin Pro 8.0 software (Microcal Software Inc., MA, USA).

In this study, we have used LSER to explain anti-oxidation activities of three polyphenols such as catechin, epicatechin and quercetin.

The general LSER equation used in this work is:

$$
\log \mathrm{A}=\mathrm{c}+\mathrm{m}\left(\frac{\mathrm{V}_{\mathrm{x}}}{100}\right)+\mathrm{s} \pi^{\mathrm{H}}+\mathrm{a} \Sigma \alpha^{\mathrm{H}}+\mathrm{b} \Sigma \beta^{\mathrm{H}}
$$

where, $\mathrm{A}$ is the find anti-oxidation activities. The $\mathrm{V}_{\mathrm{x}} \pi^{\mathrm{H}}, \Sigma \alpha^{\mathrm{H}}$ and $\Sigma \beta^{\mathrm{H}}$ terms are compound descriptors, where $\mathrm{V}_{\mathrm{x}}$ represents the compound's size/polarizability, $\pi^{\mathrm{H}}$ is the dipolarity/polarizability, $\Sigma \alpha^{\mathrm{H}}$ is the hydrogen bond (HB) acidity and $\Sigma \beta^{\mathrm{H}}$ is the hydrogen bond basicity.

The coefficients of these descriptors $\mathrm{m}, \mathrm{s}$, a and $\mathrm{b}$ reflect differences in the two bulk phases between which the solute is transferring $^{9}$ and are obtained through a multiparameter linear regression. The $\mathrm{c}$ term is simply the intercept of the regression and is comprised of constant contributions. We note that since the parameters $\mathrm{V}_{\mathrm{x}}$ and $\pi^{\mathrm{H}}$ are blends of two different interactions, the coefficients of these parameters are also blends of the corresponding properties. Specifically, $m$ is the difference in the cohesivity/dispersive ability of the two bulk phases and $\mathrm{s}$ is the difference in the ability of the two phases to interact through dipole-dipole and dipole-induced dipole interactions. Many reviews and examples of LSERs and their interpretations are available $e^{10-14}$.

\section{RESULTS AND DISCUSSION}

The antioxidation activities of the three polyphenols (catechin, epicatechin and quercetin) were examined and compared using the solvation parameter LSER model, i.e., model described in eqn. 1. The test compounds and their descriptors used in this study are given in Table-1. The descriptors of test compounds were found from reference ${ }^{15}$. Than the antioxidation activities such as ferric reducing power (FRAP) assay, two methods based on the ability to scavenge the $\mathrm{ABTS}^{-+}$radical cation, scavenging of the stable $\mathrm{DPPH}^{*}$ radical and of authentic peroxynitrite $\left(\mathrm{ONOO}^{-}\right)$of three polyphenols were listed in Table-2. These antioxidation activities were calculated which logarithms. Raw date were found from references ${ }^{2,4}$.

\begin{tabular}{|c|c|c|c|c|}
\hline \multicolumn{5}{|c|}{$\begin{array}{c}\text { TABLE-1 } \\
\text { TEST POLYPHENOLS AND THEIR DESCRIPTORS } \\
\text { FOR THE SOLVATION PARAMETER MODEL }^{15}\end{array}$} \\
\hline \multirow{2}{*}{ Compounds } & \multicolumn{4}{|c|}{ Descriptors } \\
\hline & $\mathrm{V}_{\mathrm{X}} / 100$ & $\pi^{\mathrm{H}}$ & $\alpha^{\mathrm{H}}$ & $\beta^{\mathrm{H}}$ \\
\hline Catechin & 1.99 & 2.45 & 2.30 & 1.81 \\
\hline Epicatechin & 1.99 & 73 & 2.30 & 1.84 \\
\hline Quercetin & 1.96 & 44 & 1.94 & 1.32 \\
\hline \multicolumn{5}{|c|}{$\begin{array}{c}\text { TABLE-2 } \\
\log \text { A (A: ANTIOXIDATION ACTIVITIES) } \\
\text { OF THREE POLYPHENOLS } \\
\end{array}$} \\
\hline \multirow{2}{*}{ Antioxidation } & \multicolumn{4}{|c|}{ Compounds } \\
\hline & Catechin & Epicatechin & Quercetin & Ref \\
\hline ABTS/peroxidase & 0.13 & 0.32 & 0.49 & 4 \\
\hline ABTS/persulphate & 0.58 & 0.63 & 0.83 & 4 \\
\hline FRAP assay & 0.18 & 0.13 & 0.49 & 4 \\
\hline $\mathrm{IC}_{50}\left[\mathrm{ONOO}^{-}\right](\mu \mathrm{M})$ & 1.75 & 1.75 & 1.69 & 2 \\
\hline $\mathrm{IC}_{50}\left[\mathrm{DPPH}^{*}\right](\mu \mathrm{M})$ & 0.83 & 0.83 & 0.74 & 2 \\
\hline DPPH inhibition (\%) & 1.27 & 1.27 & 1.35 & 2 \\
\hline
\end{tabular}

The coefficients for the LSER equations obtained for antioxidation activities were listed in Table- 3 and Fig. 1. The regression coefficients were above 0.99 in all of the antioxidation effects. In the ABTS/peroxidase, ABTS/persulphate, FRAP assay and DPPH inhibition, the values of $b$ were negative, it mean that an increase in the hydrogen bond basicity decreases 
TABLE-3

CONSTANTS FOR THE ANTIOXIDATION EFFECTS USING SOLVATION PARAMETER MODEL

\begin{tabular}{ccccccc}
\hline & \multicolumn{5}{c}{ Antioxidation activities } \\
\cline { 2 - 7 } & ABTS/peroxidase & ABTS/persulphate & FRAP assay & $\mathrm{IC}_{50}[\mathrm{ONOO}](\mu \mathrm{M})$ & $\mathrm{IC}_{50}\left[\mathrm{DPPH}^{*}\right](\mu \mathrm{M})$ & $\mathrm{DPPH}$ inhibition $(\%)$ \\
\hline $\mathrm{b}$ & -0.75 & -0.51 & -0.61 & 0.12 & 0.17 & -0.15 \\
$\mathrm{a}$ & 0.00 & 0.00 & 0.00 & 0.00 & 0.00 & 0.00 \\
$\mathrm{~s}$ & 0.76 & 0.22 & -0.14 & 0.01 & 0.004 & -0.01 \\
$\mathrm{~m}$ & 0.00 & 0.00 & 0.00 & 0.00 & 0.00 & 0.00 \\
$\mathrm{c}$ & -0.36 & 0.97 & 1.64 & 1.51 & 0.50 & 1.57 \\
$\mathrm{r}^{2}$ & 0.9970 & 0.9999 & 0.9990 & 0.9990 & 0.9990 & 0.9999 \\
\hline
\end{tabular}

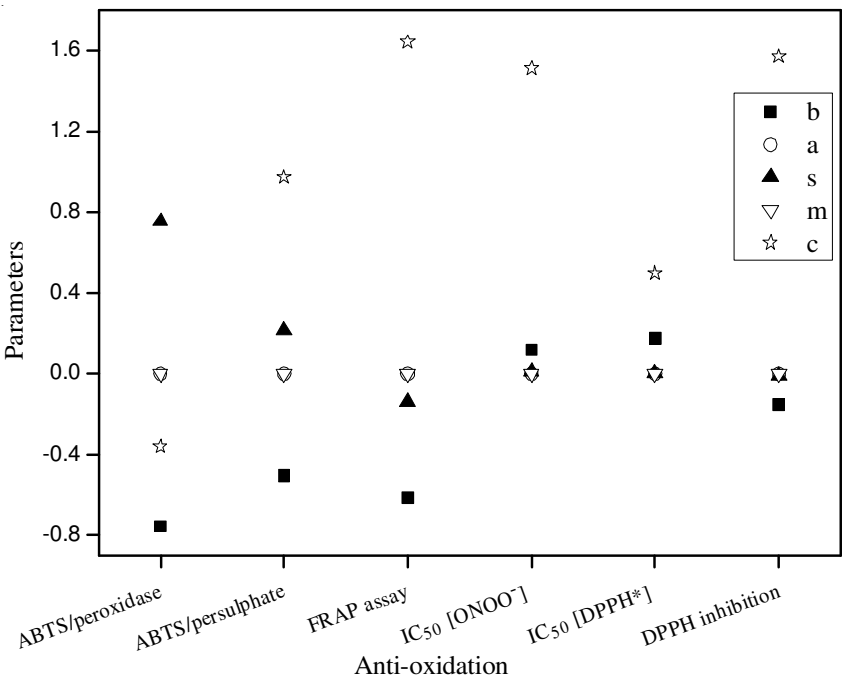

Fig. 1. LSER coefficients as a function of antioxidation activities

the antioxidation activities of the molecule. Furthermore, most of the values of s were positive in ABTS/peroxidase, ABTS/ persulphate, $\mathrm{IC}_{50}\left(\left[\mathrm{ONOO}^{-}\right]\right.$and $\left.\left[\mathrm{DPPH}^{*}\right]\right)$ studies, indicating that an increases in the dipolarity/polarizability increases the above antioxidation effects. Furthermore, all of the values of $\mathrm{m}$ and a were zero in all studies, indicating that the size/polarizability and hydrogen bond (HB) acidity were no effects for antioxidation activities of these three polyphenols. In view of value range of coefficients, the solute dipolarity/polarizability $\left(\pi^{\mathrm{H}}\right)$ generally play the largest role in determining the antioxidation effect of three polyphenols in all studies. Hydrogen bond basicity $\left(\Sigma \beta^{\mathrm{H}}\right)$ is also an important factor in the each antioxidation activities with coefficients comparable in magnitude to those three polyphenols.

The difference in dipolarity/polarizability is represented by the coefficient $\mathrm{s}$. A negative sign for this coefficient indicates that these antioxidation effects experience a microenvironment that has less dipolar/polarizable characteristics than the other antioxidation assay. The values of $s$ were negative in FRAP assay and DPPH inhibition.

The coefficient a is an important factor in the solvatochromic model in the two systems at other studied. This coefficient represents the difference in the hydrogen bond accepting basicity of the ionic liquid mobile phase and that of the aqueous phase in separation area. But in this study, all of the values of a of antioxidation effects were can not calculated. Because all of the hydrogen bond (HB) acidities of these three polyphenols were zero.

The coefficient $b$ is the most important factor in the LSER solvation parameter model used in this study. The b coeffi- cient is proportional to the difference in the hydrogen bond donating ability of the three polyphenols.

Calculated $\log$ A values of the three polyphenols were computed for antioxidation activities using eqn. 1 . The relationship between calculated (cal) and experimental (exp) log A were showed in Fig. 2. The solvation parameter model is found to provide statistically and chemically results. The correlation between experimental (exp) and calculated (cal) $\log$ A (antioxidation activities) demonstrated in Fig. 2. Almost the experimental and calculated data were on the diagonal neighborhood. This indicates that these experimental data is well fitted to the LSER models. It showed that LSERs are able to approximately reproduce the experimental $\log$ A values for the three polyphenols studied in the different antioxidation activities.

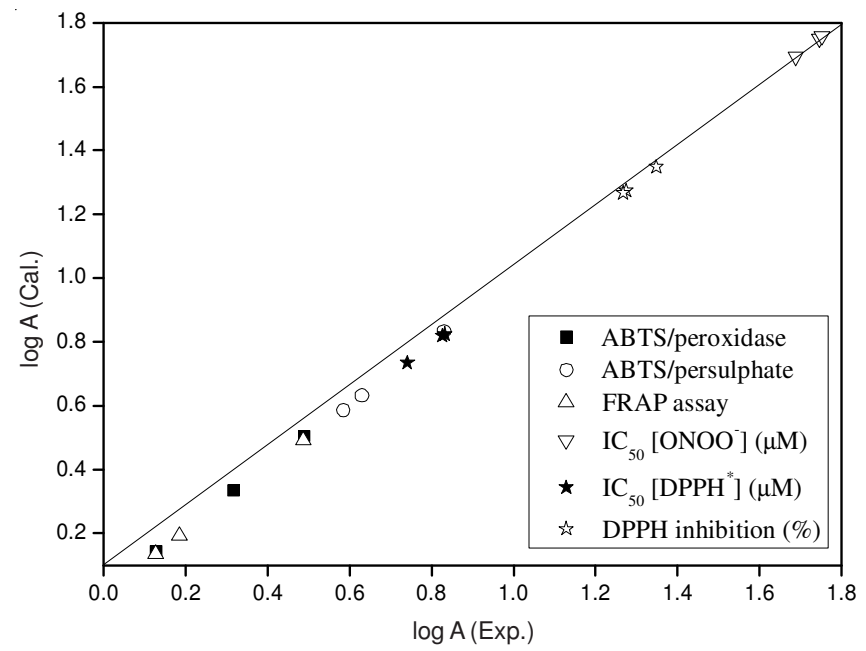

Fig. 2. Correlation between experimental (exp) and calculated (cal) $\log A$

\section{Conclusion}

Three polyphenols applied as target compounds in the anti-oxidation effects using LSER model. The LSER model, i.e., the solvation parameter model, was successfully applied to investigate the effects of the anti-oxidation of three polyphenols. The results obtained from the solvation parameter model provide comparable information, for example, coefficient $s$ and coefficient b play the most important role in antioxidation behaviour in above different experimental conditions. It is worth noting that, using the obtained LSER models, it is possible to predict antioxidation effects with high correlation coefficients $\left(r^{2}>0.99\right)$. It is evident from the results of the LSER model that the dipolarity/polarizability and hydrogen bond basicity have dominant effects role on the anti-oxidation effects 
of three polyphenols. This model is a helpful to understand the solute-organic compound interactions and evaluate the antioxidation effects of useful compounds.

\section{ACKNOWLEDGEMENTS}

This research was supported by the Science and Technology Commission of Shanghai Municipality (12290502200), Natural Science Foundation of China (51269032), Shanghai University Knowledge Service Platform and Shanghai Ocean University Aquatic Animal Breeding Center (ZFI206) and National Research Foundation (NRF) of Korea funded by the Ministry of Education, Science and Technology (No. 2011-0010673).

\section{REFERENCES}

1. Y.H. Xuan, Y. Jin, K.H. Row and Y.S. Jin, Asian J. Chem., 22, 7219 (2010).

2. P. Iacopini, M. Baldi, P. Storchi and L. Sebastiani, J. Food Comp. Anal., 21, 589 (2008)
3. B.H. Havsteen, Pharmacol. Therap., 96, 67 (2002).

4. M. Duenas, S. González-Manzano, A. González-Paramás and C. SantosBuelga, J. Pharm. Biomed. Anal., 51, 443 (2010).

5. C.A. Graffis and D.S. Ballantine, J. Chromatogr. A, 946, 185 (2002)

6. M.J. Kamlet, R.M. Doherty, M.H. Abraham, Y. Marcus and R.W. Taft, J. Phys. Chem., 92, 5244 (1988).

7. M.J. Kamlet, R.M. Doherty, P.W. Carr, D. Mackay, M.H. Abraham and R.W. Taft, Sci. Technol., 22, 503 (1988).

8. T. Wang, X. Wang and R.L. Smith Jr., J. Supercritical Fluids, 35, 18 (2005).

9. B.W. Gung, X.W. Xue and W.R. Roush, J. Am. Chem. Soc., 125, 3668 (2003).

10. P.W. Carr, R.M. Doherty, M.J. Kamlet, R.W. Taft, W. Melander and C. Horvath, Anal. Chem., 58, 2674 (1986).

11. L.C. Tan and P.W. Carr, J. Chromatogr. A, 799, 1 (1998).

12. M.H. Abraham, H.S. Chadha and A.J. Leo, J. Chromatogr. A, 685, 203 (1994).

13. J.H. Park, J.J. Chae, T.H. Nah and M.D. Jang, J. Chromatogr. A, 664, 149 (1994).

14. M. Tian, S. Li and K.H. Row, Korean J. Chem. Eng., 28, 357 (2011).

15. P. Jandera, P. Cesla, T. Hajek, G. Vohralik, K. Vynuchalova and J. Fischer, J. Chromatogr. A, 1189, 207 (2008). 